\title{
Microcamera aperture scale in monocentric gigapixel cameras
}

\author{
Daniel L. Marks, ${ }^{1}$ Eric J. Tremblay, ${ }^{2}$ Joseph E. Ford, ${ }^{2}$ and David J. Brady ${ }^{1, *}$ \\ ${ }^{1}$ Department of Electrical and Computer Engineering and Fitzpatrick Institute for Photonics, \\ Duke University, Box 90291, Durham, North Carolina 27708, USA \\ ${ }^{2}$ Department of Electrical and Computer Engineering, University of California at San Diego, \\ Engineering Building 1, 9500 Gilman Drive, La Jolla, California 92093-0407, USA \\ ${ }^{*}$ Corresponding author: dbrady@ee.duke.edu \\ Received 23 May 2011; revised 22 July 2011; accepted 16 August 2011; \\ posted 18 August 2011 (Doc. ID 147990); published 14 October 2011
}

\begin{abstract}
Multiscale cameras achieve wide-angle, high-resolution imaging by combining coarse image formation by a simplified wide-field objective with localized aberration correction in an array of narrow field microcameras. Microcamera aperture size is a critical parameter in multiscale design; a larger aperture has greater capacity to correct aberration but requires a more complex microcamera optic. A smaller aperture requires integration of more microcameras to cover the field. This paper analyzes multiscale system performance as a function of microcamera aperture for 2 and 40 gigapixel monocentric objective lenses. We find that microcamera aperture diameters of 3 to $12 \mathrm{~mm}$ paired with complementary metal oxide semiconductor sensors in the 1 to 15 megapixel range are most attractive for gigapixel-scale cameras. (C) 2011 Optical Society of America
\end{abstract}

OCIS codes: $\quad 220.4830,220.1000,220.3620,220.1250$.

\section{Introduction}

While space-bandwidth product analysis suggests that the pixel capacity of a camera is approximately equal to aperture area divided by wavelength squared, current cameras do not approach this limit for aperture diameters greater than $\approx 1000 \lambda$. At larger scales geometric aberration dominates diffraction unless field of view, and thus pixel capacity, is reduced [1]. Multiscale lens systems overcome this limitation by breaking the aberration correction problem into a hierarchical structure, with preliminary coarse image formation by a large aperture objective lens and secondary fine image formation in a microcamera array [2].

Previous applications of secondary lens arrays in camera systems have supported one microlens per pixel to increase fill factor [3] or a few pixels per microlens to enable plenoptic [ $\overline{4}]$ and light field imaging

0003-6935/11/305824-10\$15.00/0

(C) 2011 Optical Society of America
[5]. Microcameras in multiscale systems, in contrast, image onto relatively large segmented focal plane arrays. The ideal microcamera is as large as available focal plane technology will allow while remaining small enough to support full space-bandwidth product image capture. In practice, this means that microcamera apertures approach the $1000 \lambda$ limit of conventional diffraction-limited full-field cameras. The basic idea is that one finds the ideal balance of information capacity and lens simplicity in the microcamera and then one uses this system to build larger systems. Microcameras are similar in this regard to microprocessors; just as one builds supercomputers from microcomputer arrays, multiscale design allows one to build supercameras from microcamera arrays. And, just as the central challenge of multiprocessor design is to determine the balance between single-core and multicore capabilities, the central challenge of multiscale design is to determine the relative processing capacity of the microcamera.

Aperture size is the most significant parameter in microcamera design. A larger aperture may simplify 
camera integration by requiring fewer microcameras (to the extreme example of only one microcamera for imagers below 1000 $\lambda$ ). Larger apertures also have a larger wavefront and thus more capacity for wavefront correction. Of course, the larger aperture also introduces a greater capacity for wavefront distortion in the microcamera, which may require a more complex lens system. Smaller microcamera apertures may allow more aggressive surface forms and more integrated manufacturing technologies. Our goal in this paper is to illuminate trade-offs in microcamera aperture selection by presenting two specific multiscale design examples.

Our choice of design examples is illuminated by our previous studies of the general utility of monocentric lenses in multiscale systems [6] and of the specific capacity of the Gigagon lens [7]. Monocentric objectives are particularly attractive in multiscale design because their imaging characteristics remain invariant over a wide angular range, which is illustrated in Fig. 1. In exchange for this simplicity, relatively few variables are available for aberration correction in monocentric lenses. Significant microcamera aberration correction is required, particularly as objective scale increases.

The complexity of the microcamera depends on the type and magnitude of aberrations it is intended to correct. The relative magnitude of various aberrations of objectives for 1-5 gigapixel imaging is different than from objectives intended for 20-40 gigapixel imaging with a corresponding difference in microcamera design. Microcamera design is also impacted by vignetting, aperture packing, and focal plane integration issues [8]. Microcameras must be substantially faster than the objective to avoid significant power loss and aberration due to vignetting.

With these trade-offs in mind, this paper presents specific examples of optical system performance for two Gigagon lenses: one designed with 2 gigapixel full-field capacity and one with 40 gigapixel capacity. The design and performance of the Gigagon lenses is described in Section 2 . Section 3 describes the performance of each system over a range of microcamera apertures. In the end, just as fairly standard microprocessor scales have emerged for computers spanning a wide range of system performance, we

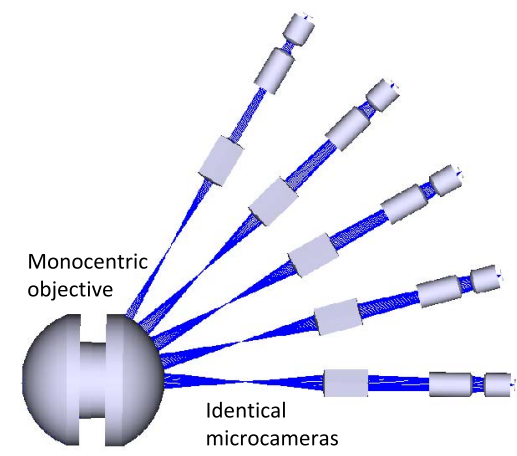

Fig. 1. (Color online) Shaded model of an objective lens with several microcameras. anticipate that microcamera architectures will settle on reasonable $1-10 \mathrm{~mm}$ system scales compatible with full space-bandwidth integration over a large range of systems. We summarize our conclusions in this regard in Section $\underline{4}$.

\section{Monocentric Objectives for Multiscale Designs}

For this study, two objectives were designed: one for 2 gigapixel imaging and another for 40 gigapixels. The number of gigapixels of each objective was calculated assuming that, at the focal surface, the largest usable spatial frequency on the focal surface of each objective is 200 cycles $/ \mathrm{mm}$. As will be seen, the 2 gigapixel objective can directly form an image onto its focal surface with 200 cycles/mm resolution; however, the 40 gigapixel camera requires microcamera correction to reach this limit of resolution. For each of these objectives, microcameras were designed with aperture sizes $0.375,0.75,1.5,3,6,9,18$, and $36 \mathrm{~mm}$, and the performance of these microcameras are compared for the two objectives. We first examine the two monocentric objectives and their designs to determine how their performance is limited by aberrations. The two size scales are chosen to exemplify the aberrations that are present in the objective for small and large aperture monocentric objectives.

The described monocentric objectives consist of a spherical core of a crown glass (low index and high Abbe number) surrounded by meniscus shells of flint glasses (high index and low Abbe number) with the shell surfaces concentric with the spherical core center. A stop is placed at the center of curvature to limit the $f$ /\#. Excluding the vignetting caused by the stop, the lens is symmetric with the respective incoming field angle. Unlike an ordinary lens, monocentric systems have no tightly defined optical axis. This means that no off-axis aberrations, e.g., lateral chromatic aberration, astigmatism, or coma, are present. The only aberrations present are axial chromatic aberration, spherical aberration, and spherochromatism. In addition, the image has field curvature because it is formed on a spherical surface also concentric with the core. In designing the microcameras, monocentric objectives are especially attractive because the objective aberrations are independent of field angle, and therefore the same microcamera design corrects the aberrations at all field positions with respect to the objective.

To understand how aberrations change as the objective size scales, we consider how the optical path difference of an optical system scales as the entire optical system scales in size (all radius of curvatures and lengths, except for the wavelength of illumination). For example, an optical system that satisfies the Rayleigh criterion of a peak-to-valley quarterwave error at a particular scale does not satisfy the criterion when the optics are doubled in size because the new peak-to-valley error is now a half wave. If an optical system is scaled to be very small, its optical path difference error becomes correspondingly less, and therefore the image quality improves. 

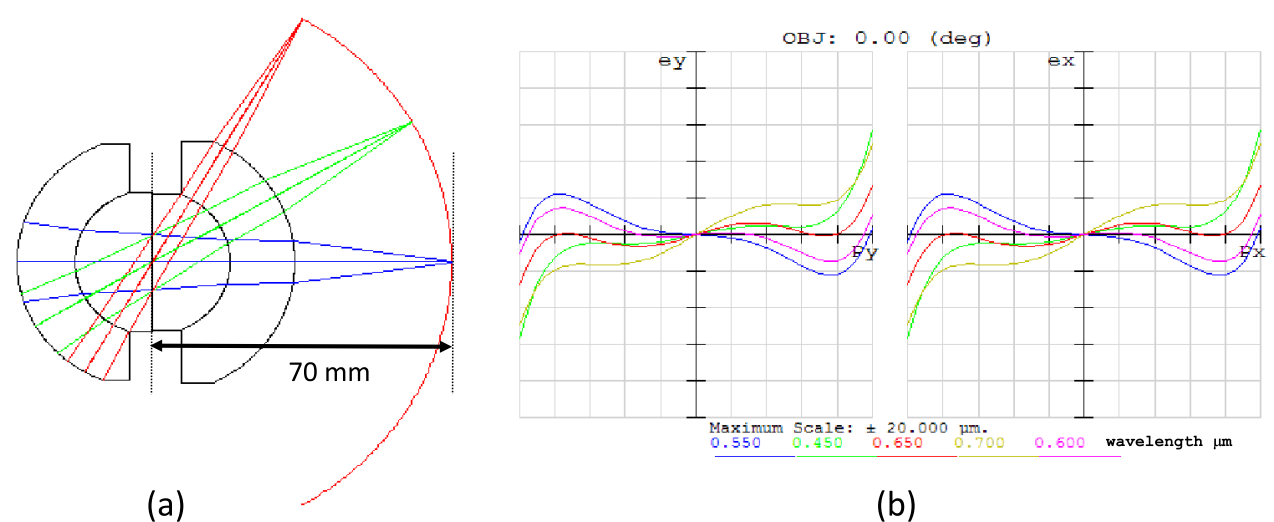

(a)

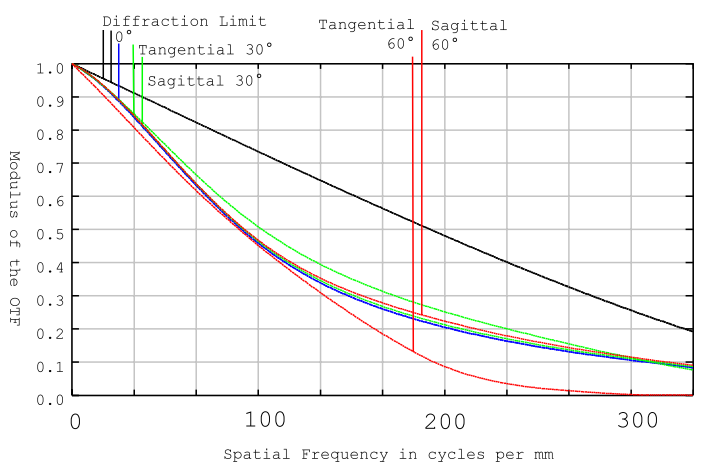

(b)

(c)

Fig. 2. (Color online) Specifications of the 2 gigapixel objective. (a) Diagram and ray trace of the lens. (b) Ray fan plot. (c) Modulation transfer function curve.

The size of the image also correspondingly decreases. With a monocentric design, the image is formed on a surface concentric with the center of curvature, and the radius of this image also decreases with decreasing scale. Therefore, while chromatic and spherical aberration become less prominent at smaller scales, curvature of field becomes more important. The opposite is true at larger scales, with chromatic and spherical aberrations becoming more difficult to correct than the curvature of field. It is the relative magnitude of these aberrations that limits the performance of a particular microcamera and objective system.

As an example of a smaller objective for which field curvature dominates, a 2 gigapixel objective is shown in Fig. 2, with its prescription described in Table $\underline{1}$, with the glasses being found in the Ohara catalog (Ohara Corp., Branchburg, New Jersey). In this figure and succeeding figures, OTF refers to the optical transfer function. The design wavelengths are from
480 to $640 \mathrm{~nm}$, and the $f / \#$ is 3.5 with a focal length and image curvature radius of $70.256 \mathrm{~mm}$. Figure 2 (a) is a diagram of the lens with ray traces for the field angles $0^{\circ}, 20^{\circ}, 40^{\circ}$, and $60^{\circ}$. Figure $2(\mathrm{~b})$ is a ray fan plot for the $0^{\circ}$ fan (the higher angle fans are partially vignetted but otherwise identical). The ray fan shows partially corrected spherical aberrations with a comparable amount of focus error due to chromatic aberration. The modulation transfer function of Fig. 2(c) is favorable, and the lens can achieve fairly good performance out to 200 cycles $/ \mathrm{mm}$. Because the quality of the image formed by the objective is already good, the primary purpose of the microcamera is to relay a portion of the curved field to its respective sensor.

In contrast to the 2 gigapixel objective, the 40 gigapixel objective of Fig. 3 has more chromatic and spherical aberrations. The prescription is listed in Table 2. It is designed only for the wavelengths 500 to $600 \mathrm{~nm}$ and is $f / 3.0$ with a focal length and image radius of curvature of $299.765 \mathrm{~mm}$. The 40 gigapixel

Table 1. Prescription for the 2 Gigapixel Microcamera Objective

\begin{tabular}{cccl}
\hline Comments & Radius of Curvature $(\mathrm{mm})$ & Thickness $(\mathrm{mm})$ & \multicolumn{1}{c}{ Glass Type } \\
\hline Object surface & $\infty$ & $\infty$ & \\
& 31.800 & 13.613 & S-NBH8 $\left(n_{d}=1.720467, V_{d}=34.707984\right)$ \\
Stop & 18.187 & 18.187 & Fused silica $\left(n_{d}=1.458464, V_{d}=67.821433\right)$ \\
& $\infty$ & 18.187 & Fused silica $\left(n_{d}=1.458464, V_{d}=67.821433\right)$ \\
Image surface & -18.187 & 15.191 & S-NBH8 $\left(n_{d}=1.720467, V_{d}=34.707984\right)$ \\
& -33.378 & 36.877 & \\
\hline
\end{tabular}




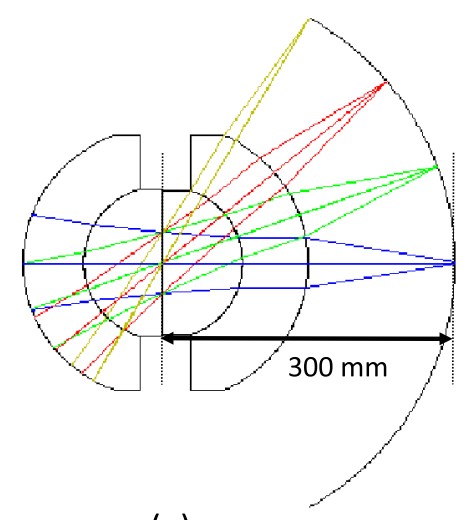

(a)

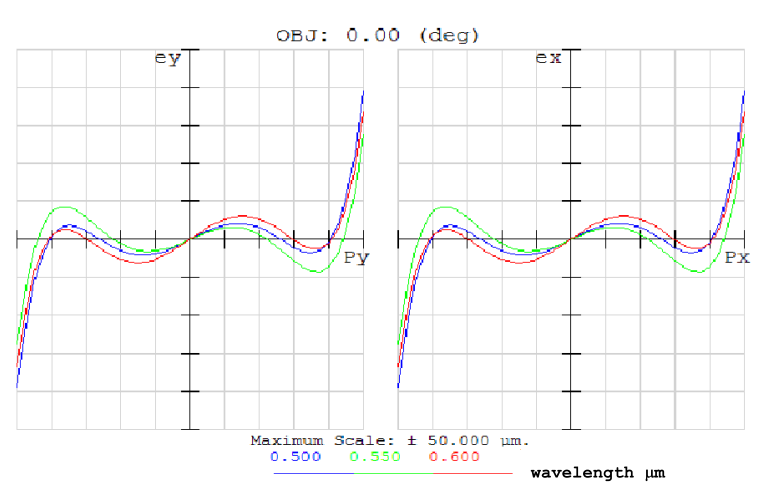

(b)

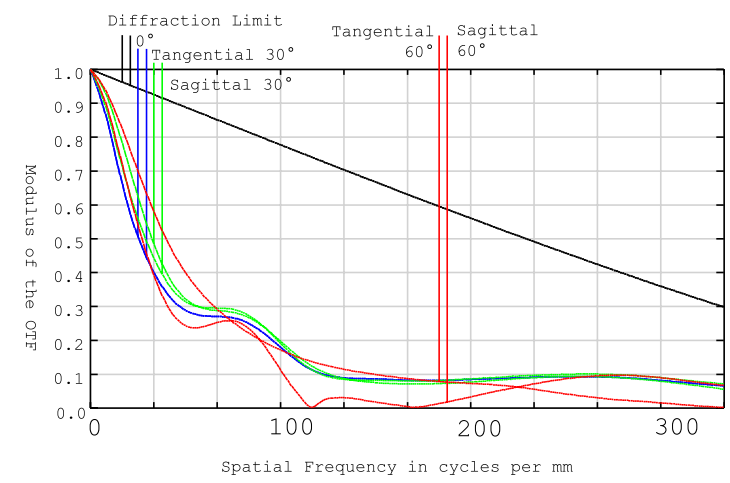

(c)

Fig. 3. (Color online) Specifications of the 40 gigapixel objective. (a) Diagram and ray trace of the lens. (b) Ray fan plot. (c) Modulation transfer function curve.

objective has a smaller $f$ /\# than the 2 gigapixel objective because the core of the 40 gigapixel objective is made of BSL7 (similar to Schott BK7), which has a larger refractive index than the fused silica core of the 2 gigapixel objective. This choice was made because larger spheres of homogeneous borosilicate glass are more readily available than fused silica. The microcamera is used to correct the chromatic aberration throughout the entire range of 486 to $656 \mathrm{~nm}$, compensating for the limited chromatic correction of the objective. Examining the ray fan plot of Fig. 3(b), it is clear that this design has significant uncorrected spherical aberration. While this lens has been achromatically corrected between 500 and $600 \mathrm{~nm}$ wavelengths, the secondary chromatic aberration would have been too great if the entire visible spectrum were corrected; therefore, it is desirable to correct the remaining chromatic error using the microcameras. The effects of these aberrations are apparent in the modulation transfer function plot of Fig. 3(c), which is calculated for the entire range from 486 to $656 \mathrm{~nm}$. The performance is significantly worse than the 2 gigapixel lens and demonstrates the necessity of microcamera field correction to achieve satisfactory imaging performance. Because the radius of curvature of the image is more than four times larger, the need for curvature-of-field correction in the microcamera is less, and therefore larger microcameras may be used to offset the increased chromatic and spherical aberrations.

\section{Microcameras}

With the prescriptions and imaging properties of the objectives established, corresponding microcameras are designed to form high-quality images from the imperfect intermediate objective images. These

Table 2. Prescription for the 40 Gigapixel Microcamera Objective

\begin{tabular}{cccc}
\hline Comments & Radius of Curvature $(\mathrm{mm})$ & Thickness $(\mathrm{mm})$ & Glass Type \\
\hline Object surface & $\infty$ & $\infty$ & \\
& 144.113 & 62.426 & LAH54 $\left(n_{d}=1.815543, V_{d}=44.361146\right)$ \\
Stop & 81.687 & 81.687 & BSL7 $\left(n_{d}=1.516630, V_{d}=64.150145\right)$ \\
& $\infty$ & 81.687 & BSL7 $\left(n_{d}=1.516630, V_{d}=64.150145\right)$ \\
Image surface & -81.687 & 67.226 & L-NBH54 $\left(n_{d}=1.902000, V_{d}=25.101413\right)$ \\
& -148.913 & 150.852 & \\
\hline
\end{tabular}


microcameras are designed with common properties to make their comparison easier and differ chiefly in their aperture size and the number of optical elements. The microcamera elements have even aspheric surfaces and are composed of either the "crown" cyclo-olefin plastic Zeonex E48R $\left(n_{d}=1.531160\right.$, $V_{d}=56.043828$, Zeon Chemicals, L.P., Louisville, Kentucky), or the "flint" plastic polycarbonate $\left(n_{d}=1.585470, V_{d}=29.909185\right)$. Plastics were used rather than optical glasses because it is expected that the complex element surfaces will be reproduced using economical plastic molding techniques. An even-polynomial-phase single-order diffractive surface was placed on an optic to aid with chromatic aberration correction and is as easily reproduced using plastic molding techniques as the aspheric surface onto which it is formed. The range of chromatic correction of the overall system (objective and microcamera) is between 480 and $640 \mathrm{~nm}$, even if the parent objective does not by itself correct throughout this range. While losses from a finite diffraction efficiency are expected [9] when used with broadband illumination, the $30 \%$ fractional bandwidth has a minimum theoretical loss of $2.5 \%$. Because diffractive elements are able to correct a large amount of chromatic aberration and chromatic aberration often limits the performance of these designs, the diffraction efficiency losses were deemed acceptable given the great benefits.

These microcameras are designed for an image $f$ /\# of 1.3, suitable for photosensor arrays with pixels from 1.25 to $1.75 \mu \mathrm{m}$ in size with the possibility of retaining a modulation transfer function magnitude of 0.5 or greater at the Nyquist sampling frequency. Such pixel sizes are common in recent generation complementary metal oxide semiconductor (CMOS) photosensor arrays intended for compact cameras and mobile phone use. The microcameras are designed to demagnify the image (with a magnification between 0.4 and 0.5 ) to reserve space between the photosensors for the mechanical mounting hardware needed to hold the sensor and microcameras together. The array size is assumed to scale with the size of the microcamera and be covered with a $0.4 \mathrm{~mm}$ thickness BSL7 window, except for microcameras with apertures $0.375 \mathrm{~mm}$ or less in diameter. As the microcamera aperture is decreased, more microcameras are needed to cover the full angular field of the objective. Inevitably there are engineering challenges associated with the mechanical and electronic integration of the perhaps hundreds or thousands of microcameras relaying the image from a gigapixel objective; however, in this work chiefly the optical performance of the microcameras is studied.

We note that, while the microcameras image an object field with $f / 3.0$ to $f / 3.5$ to $f / 1.3$, radiance is conserved. The sensors on the microcameras are significantly smaller than the field relayed by the microcamera with a magnification of approximately 0.4 . The total sensor area of all of the microcameras is a fraction of the area on the objective image surface that is relayed due to this demagnification. Furthermore, the microcameras are placed side by side with some of the rays from particular object points being split between the entrance apertures of adjacent microcameras, as explained in our earlier work [8]. Therefore, while vignetting occurs at the entrance of these microcamera apertures, this is intentional to allow multiple images of the same point to be formed on different cameras. No relay or field lens is required because the microcamera is a relay lens that is designed to vignette the rays to enable partially overlapping imaged fields.

Twelve microcamera designs for the 2 gigapixel objective are shown in Fig. 4, labeled by their aperture size. The elements are labeled by "C" for a crown plastic element, an " $\mathrm{F}$ " for the flint plastic element, and " $D$ " next to the diffractive surface. The on-axis distance from the first element to the image plane is also labeled. The corresponding performance data for these designs are documented in Table 3 . The field of view is given as the angular range of the objective field that is relayed to the microcamera image plane. This angle maintains the same number of resolvable pixels on each sensor given the scale of the microcamera, so that the same size aperture microcamera resolves a smaller field of view when used with an objective resolving more gigapixels. In most cases the field of view of a microcamera used with the 2 gigapixel objective is 4.6 times higher than the corresponding microcamera used with the 40 gigapixel objective, except when the microcamera sizes becomes a significant fraction of the objective size. Such a criterion fairly compares the performance of the microcamera given different objectives as the diameters of the relayed object fields are then similar.

The performance of the smaller microcameras, with apertures under $3 \mathrm{~mm}$ in size, is excellent. This is because smaller cameras can be placed to better conform to the curved field of the objective, so this aberration becomes less problematic with smaller microcamera size. On the other hand, the amount of chromatic and spherical aberration that the microcamera needs to correct is small, so the smaller microcameras are able to sufficiently do so. As the microcamera aperture increases in size, the primary limitation of image quality is the aberrations introduced by the microcamera optics, so more elements are needed to properly relay and demagnify the field as the aperture size increases. Therefore, for this objective the choice of aperture size is largely determined by the available focal plane sizes and the requirements of mechanical and electronic integration and not the image quality.

An additional 12 microcamera designs for the 40 gigapixel objective are shown in Fig. 5. The conventions used are the same as those for Fig. 4. Likewise, the corresponding performance data are shown in Table 4 . Unlike the case of the 2 gigapixel objective, microcameras with very small apertures (under $3 \mathrm{~mm}$ ) have poorer performance than larger 

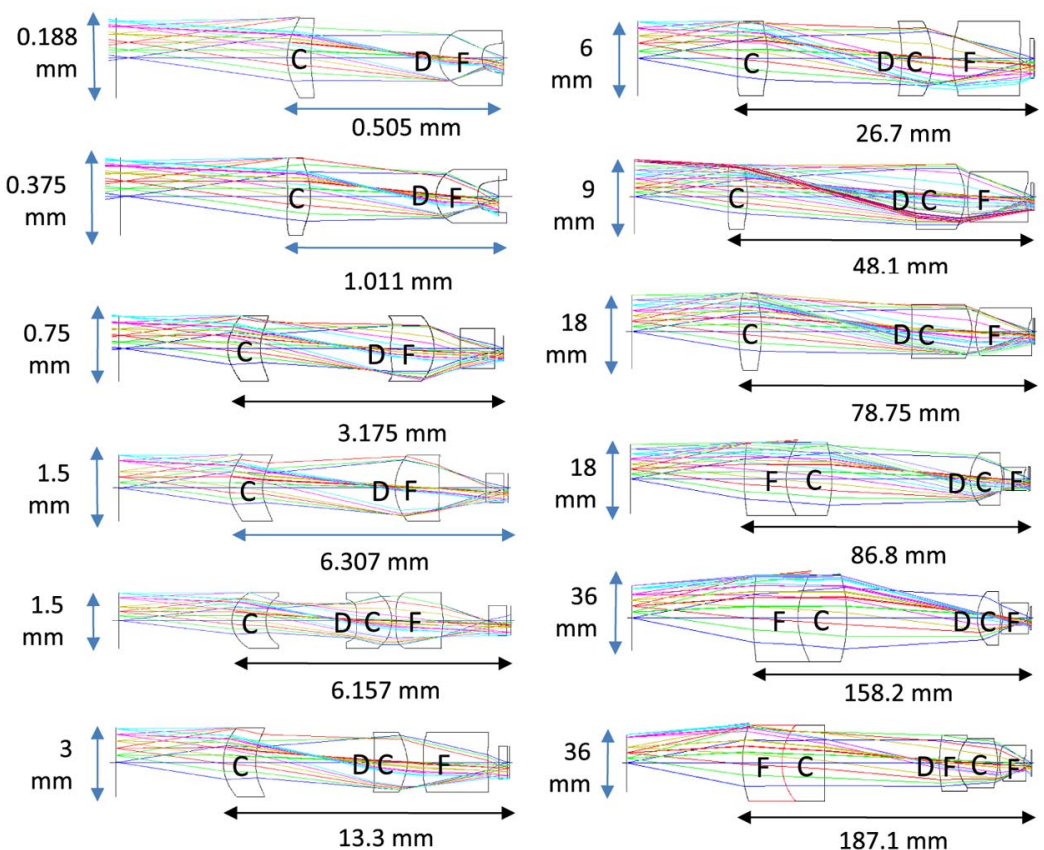

Fig. 4. (Color online) Ray traces of microcameras for the 2 gigapixel objective of various scales. A "C" indicates a crown plastic element, an "F" indicates a flint plastic element, and a " $D$ " indicates a diffractive surface.

apertures. This is because the curvature of field of the 40 gigapixel objective is correspondingly less, making the advantage of conforming to the curved field less useful, while the amount of chromatic and spherical aberration needed to be corrected has been increased. For 3 to $9 \mathrm{~mm}$ aperture microcameras, the three-element lens can perform quite well, similar to or better than the microcameras of the same aperture size for the 2 gigapixel objective. This is likely because less field curvature needs to be corrected for the 40 gigapixel lens over a single microcamera aperture compared to the 2 gigapixel objective, which allows additional freedom to choose the powers of the surfaces to correct the chromatic and spherical aberrations. We note that the $0.188 \mathrm{~mm}$ aperture is not included in Table 4 as it was in Table 3 . This is because it was difficult to obtain a reliable performance estimate for this microcamera and objective size ratio (such a system would contain millions of microcameras to cover a $120^{\circ}$ field of view). Furthermore, the case of an $18 \mathrm{~mm}$ aperture and five elements was modeled for the 40 gigapixel lens but not for the 2 gigapixel lens. This is because the four-element design for the 40 gigapixel lens has poor off-axis performance, so the five-element design was included as a successful example at that scale. A 2 gigapixel, $18 \mathrm{~mm}$ aperture, five-element design not shown works equally as well.

For the largest $36 \mathrm{~mm}$ aperture sizes, the performance is poorer than the corresponding 2 gigapixel objective $36 \mathrm{~mm}$ aperture microcameras. The difference is attributed to significantly greater chromatic aberration in the 40 gigapixel objective. As the microcamera scale increases, increased focus error from uncompensated chromatic aberration likewise increases. The chromatic focal shift of both $36 \mathrm{~mm}$ aperture, five-element microcameras is shown in Fig. $\underline{6}$. The main control over chromatic aberration

Table 3. Modulation Transfer Function of Microcameras for Various Aperture Sizes and Elements with the 2 Gigapixel Objective

\begin{tabular}{|c|c|c|c|c|c|c|c|c|}
\hline $\begin{array}{l}\text { Aperture } \\
\text { Size }(\mathrm{mm})\end{array}$ & $\begin{array}{l}\text { Number of } \\
\text { Elements }\end{array}$ & $\begin{array}{l}\text { Field of View } \\
\text { (degrees) }\end{array}$ & $\begin{array}{l}\text { MTF On-Axis } \\
150 \mathrm{~mm}^{-1}\end{array}$ & $\begin{array}{l}\text { MTF Tan Half- } \\
\text { Field } 150 \mathrm{~mm}^{-1}\end{array}$ & $\begin{array}{l}\text { MTF Sag Half- } \\
\text { Field } 150 \mathrm{~mm}^{-1}\end{array}$ & $\begin{array}{l}\text { MTF On-Axis } \\
300 \mathrm{~mm}^{-1}\end{array}$ & $\begin{array}{l}\text { MTF Tan Half- } \\
\text { Field } 300 \mathrm{~mm}^{-1}\end{array}$ & $\begin{array}{l}\text { MTF Sag Half- } \\
\text { Field } 300 \mathrm{~mm}^{-1}\end{array}$ \\
\hline 0.188 & 2 & 0.14 & 0.834 & 0.796 & 0.827 & 0.667 & 0.598 & 0.655 \\
\hline 0.375 & 2 & 0.28 & 0.823 & 0.745 & 0.816 & 0.664 & 0.506 & 0.636 \\
\hline 0.75 & 2 & 0.6 & 0.791 & 0.772 & 0.824 & 0.618 & 0.519 & 0.659 \\
\hline 1.5 & 2 & 1.2 & 0.734 & 0.608 & 0.733 & 0.517 & 0.270 & 0.513 \\
\hline 1.5 & 3 & 1.2 & 0.772 & 0.631 & 0.748 & 0.603 & 0.331 & 0.543 \\
\hline 3 & 3 & 2.4 & 0.778 & 0.664 & 0.761 & 0.589 & 0.404 & 0.560 \\
\hline 6 & 3 & 4.8 & 0.618 & 0.526 & 0.598 & 0.294 & 0.287 & 0.344 \\
\hline 9 & 3 & 8.4 & 0.406 & 0.459 & 0.404 & 0.054 & 0.217 & 0.153 \\
\hline 18 & 3 & 14 & 0.123 & 0.218 & 0.173 & 0.026 & 0.066 & 0.156 \\
\hline 18 & 4 & 14 & 0.754 & 0.602 & 0.670 & 0.540 & 0.367 & 0.402 \\
\hline 36 & 4 & 22 & 0.766 & 0.455 & 0.612 & 0.532 & 0.214 & 0.218 \\
\hline 36 & 5 & 22 & 0.840 & 0.729 & 0.802 & 0.677 & 0.485 & 0.613 \\
\hline
\end{tabular}



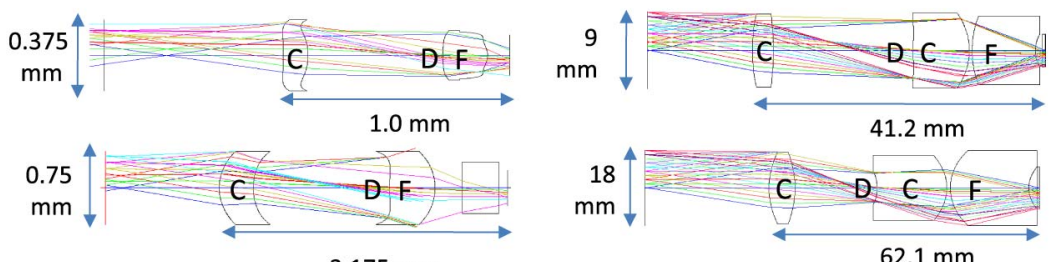

$3.175 \mathrm{~mm}$
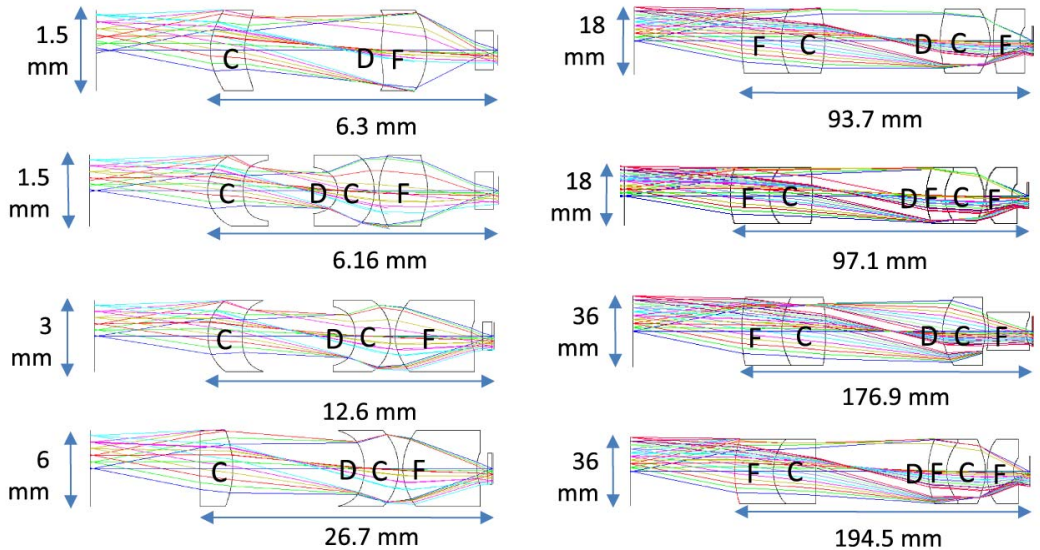

Fig. 5. (Color online) Ray traces of microcameras for the 40 gigapixel objective of various scales. A "C" indicates a crown plastic element, an "F" indicates a flint plastic element, and a "D" indicates a diffractive surface.

in these designs is the choice of materials, which are plastics, the power of the surfaces, and the diffractive surface power. Unfortunately plastics have a much smaller range of partial dispersion profiles than optical glasses do, so this means of control is not available. The diffractive element can partially compensate for this deficiency. Because the larger objective produces a correspondingly larger amount of uncorrected secondary chromatic aberration, which can be only imperfectly corrected by the microcamera, the residual chromatic aberration is also larger and therefore degrades the image quality more as the microcamera aperture size increases.

On the other hand, the $9 \mathrm{~mm}$ aperture, threeelement microcamera for the 2 gigapixel objective has significantly worse performance than its 40 gigapixel counterpart. This is also due to chromatic aberration, with the chromatic focal shift shown in Fig. 7. The microcamera for the 2 gigapixel objective has significantly more field curvature to correct. The Petzval sum, which is related to the field curvature of the objective field when the microcamera images in reverse (from focal plane to objective field), varies inversely in linear proportion to microcamera scale. For a small microcamera, the field curvature of the objective is more easily matched. However, with only a limited number of surfaces under control, at a large enough scale, both the curvature and chromatic aberration cannot be simultaneously corrected. This can be seen by examining the 3,6 , and $9 \mathrm{~mm}$ aperture three-element cameras in Fig. 4 . The surface on which the diffractive is placed (next to the letter " $D$ ") is concave for the 3 and $6 \mathrm{~mm}$ aperture microcameras, but convex in the $9 \mathrm{~mm}$ aperture microcamera. This can be compared to the $9 \mathrm{~mm}$, three-element microcamera for the 40 gigapixel objective, which has a largely flat diffractive surface. By splitting the crown element nearest the objective into an achromatic pair, the

Table 4. Modulation Transfer Function of Microcameras for Various Aperture Sizes and Elements with the 40 Gigapixel Objective

\begin{tabular}{|c|c|c|c|c|c|c|c|c|}
\hline $\begin{array}{l}\text { Aperture } \\
\text { Size }(\mathrm{mm})\end{array}$ & $\begin{array}{l}\text { Number of } \\
\text { Elements }\end{array}$ & $\begin{array}{l}\text { Field of View } \\
\text { (degrees) }\end{array}$ & $\begin{array}{c}\text { MTF On-Axis } \\
150 \mathrm{~mm}^{-1}\end{array}$ & $\begin{array}{l}\text { MTF Tan Half- } \\
\text { Field } 150 \mathrm{~mm}^{-1}\end{array}$ & $\begin{array}{l}\text { MTF Sag Half- } \\
\text { Field } 150 \mathrm{~mm}^{-1}\end{array}$ & $\begin{array}{c}\text { MTF On-Axis } \\
300 \mathrm{~mm}^{-1}\end{array}$ & $\begin{array}{l}\text { MTF Tan Half- } \\
\text { Field } 300 \mathrm{~mm}^{-1}\end{array}$ & $\begin{array}{l}\text { MTF Sag Half- } \\
\text { Field } 300 \mathrm{~mm}^{-1}\end{array}$ \\
\hline 0.375 & 2 & 0.0625 & 0.559 & 0.390 & 0.461 & 0.307 & 0.221 & 0.224 \\
\hline 0.75 & 2 & 0.125 & 0.607 & 0.605 & 0.580 & 0.281 & 0.344 & 0.271 \\
\hline 1.5 & 2 & 0.25 & 0.497 & 0.540 & 0.613 & 0.295 & 0.257 & 0.298 \\
\hline 1.5 & 3 & 0.25 & 0.729 & 0.609 & 0.692 & 0.471 & 0.328 & 0.387 \\
\hline 3 & 3 & 0.5 & 0.764 & 0.694 & 0.745 & 0.560 & 0.432 & 0.519 \\
\hline 6 & 3 & 1.0 & 0.758 & 0.715 & 0.754 & 0.560 & 0.477 & 0.559 \\
\hline 9 & 3 & 1.8 & 0.824 & 0.727 & 0.798 & 0.646 & 0.479 & 0.603 \\
\hline 18 & 3 & 3.6 & 0.447 & 0.297 & 0.396 & 0.189 & 0.106 & 0.202 \\
\hline 18 & 4 & 3.6 & 0.747 & 0.466 & 0.675 & 0.519 & 0.172 & 0.429 \\
\hline 18 & 5 & 3.6 & 0.800 & 0.622 & 0.764 & 0.592 & 0.352 & 0.569 \\
\hline 36 & 4 & 7.2 & 0.598 & 0.455 & 0.253 & 0.365 & 0.068 & 0.202 \\
\hline 36 & 5 & 7.2 & 0.708 & 0.317 & 0.579 & 0.426 & 0.087 & 0.352 \\
\hline
\end{tabular}




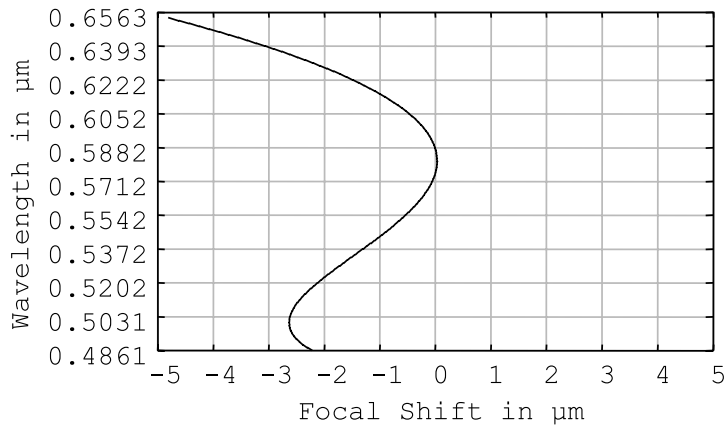

(a)

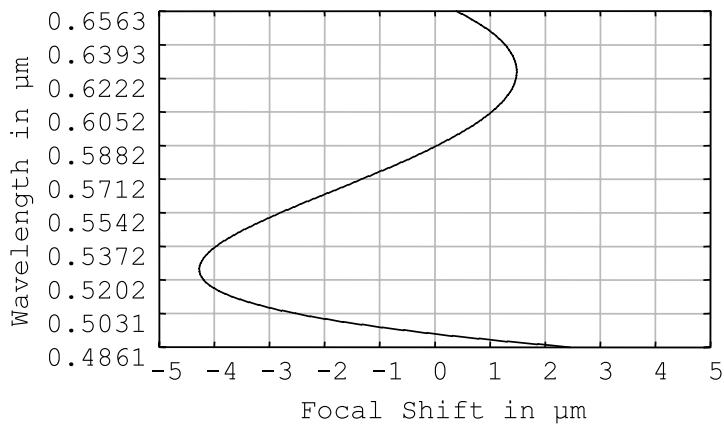

(b)

Fig. 6. Change in focus with wavelength for the $36 \mathrm{~mm}$ aperture, five-element microcameras. (a) Chromatic focal shift of 2 gigapixel objective microcamera. (b) Chromatic focal shift of the 40 gigapixel objective microcamera.

additional control is gained to decouple the field curvature and chromatic aberrations.

Ray fans of the $9 \mathrm{~mm}$ aperture, three-element microcameras are presented in Figs. 8 and 9 . As can be seen, the 2 gigapixel microcamera is chromatic aberration limited due to its widely separated ray fans for each color. This is despite the excellent chromatic correction and spherical aberration correction of the 2 gigapixel objective, showing that correcting field curvature of the microcamera object field can be a significant burden if too few elements are used. On the other hand, the 40 gigapixel microcamera is able to achieve high performance because of the comparatively small field curvature given by the inverse of the focal length of the objective. A magnitude of the optical transfer function (MTF) plot of both microcameras is also included to show the imaging performance more directly.

The most useful microcamera aperture scales are likely to be between 3 and $18 \mathrm{~mm}$. Smaller than $3 \mathrm{~mm}$ microcameras tend to work poorly for larger objectives, and larger than $18 \mathrm{~mm}$ chromatic compensation becomes increasingly difficult. This scale is well matched to the size of CMOS focal plane arrays currently in production for digital cameras and mobile phones. Three-element microcameras are likely to be sufficient for 3-6 mm apertures, four elements are needed for $6-18 \mathrm{~mm}$ apertures, with five elements sometimes being needed for apertures $10-20 \mathrm{~mm}$ in size when the objective is larger. The choice between these scales will depend on other factors such as mechanical and electronic integration and image stitching computational burden. The elements in these microcamera designs have been grouped to minimize the burden of placement of these groups and therefore the possibility of alignment error.

The alignment tolerances between the objective and the microcameras tend to be generous if a focus compensator for the sensor is used. As an example, we consider both $18 \mathrm{~mm}$ four-element designs, both of which produce reasonable performance. For the 2 gigapixel objective, decenters between the axis of the objective and the microcamera can be quite large, up to $\pm 0.3 \mathrm{~mm}$ with less than $10 \%$ increase in rootmean-square (RMS) wavefront variation. The tilt tolerance is up to $\pm 0.1^{\circ}$ with a $10 \%$ increase in RMS wavefront variation. The axial positioning tolerance is consistent with the depth of focus of the objective image of approximately $\pm 50 \mu \mathrm{m}$ for a $10 \%$ increase in RMS wavefront error. For the 40 gigapixel objective, the decenter tolerances are similarly insensitive, likely because of the decreased curvature of field of the objective image. A similar $\pm 0.06^{\circ}$ tilt tolerance produces a $10 \%$ increase in RMS wavefront variation for the 10 gigapixel objective microcamera. There is a

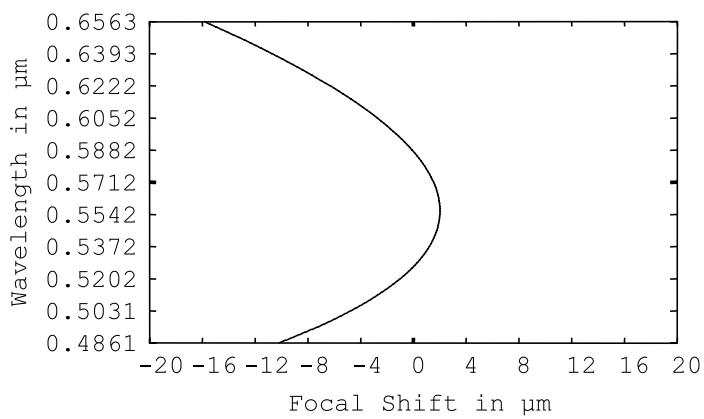

(a)

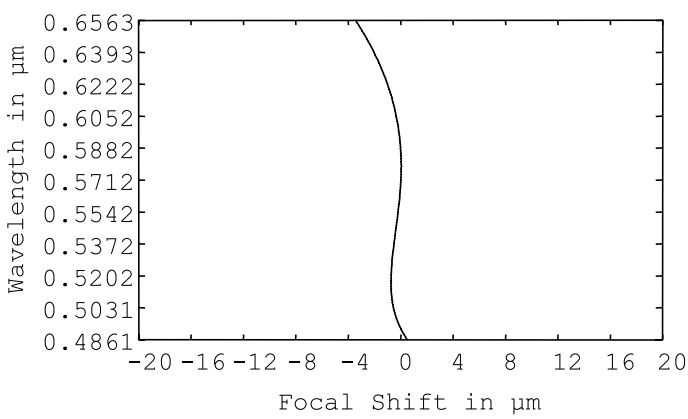

(b)

Fig. 7. Change in focus with wavelength for the $9 \mathrm{~mm}$ aperture, three-element microcameras. (a) Chromatic focal shift of 2 gigapixel objective microcamera. (b) Chromatic focal shift of the 40 gigapixel objective microcamera. 


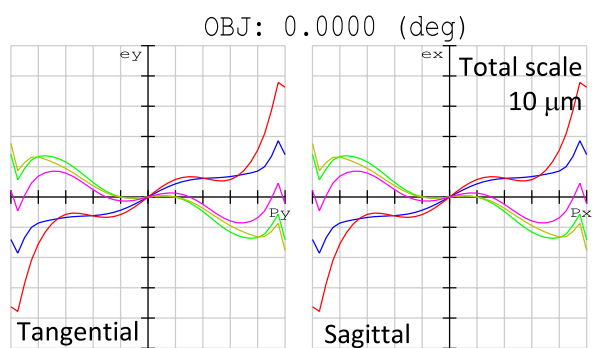

(a)

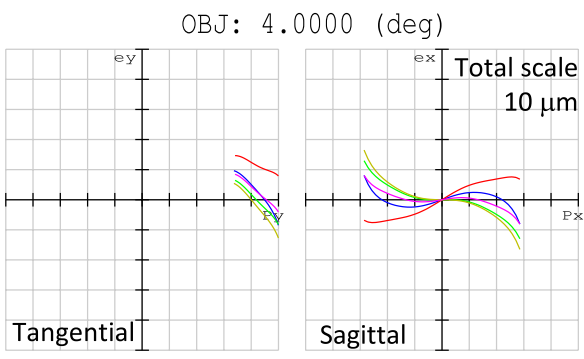

(c)

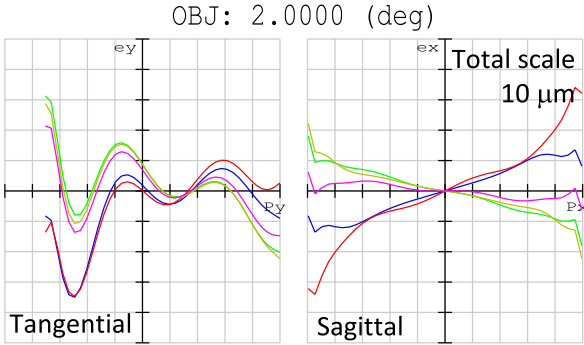

(b)

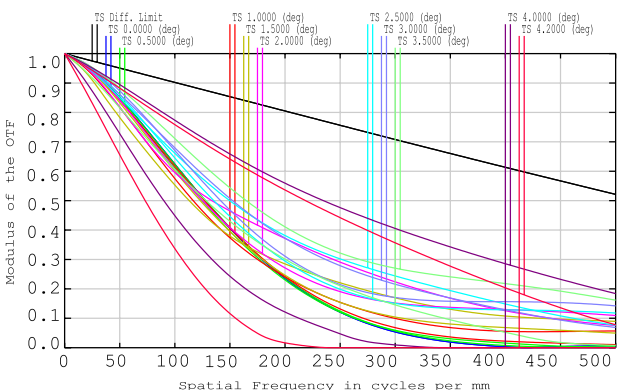

(d)

Fig. 8. (Color online) Ray traces and MTF of $9 \mathrm{~mm}$ aperture, three-element microcamera for the 2 gigapixel objective. (a), (b), and (c) Ray traces for on-axis, half-field, and full-field object points, respectively. (d) Sagittal and tangential modulation transfer functions of this camera as a function of object point. T denotes tangential curves and S denotes sagittal curves in the MTF plot.

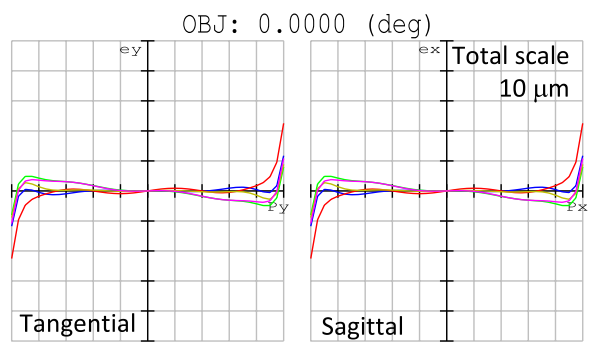

(a)

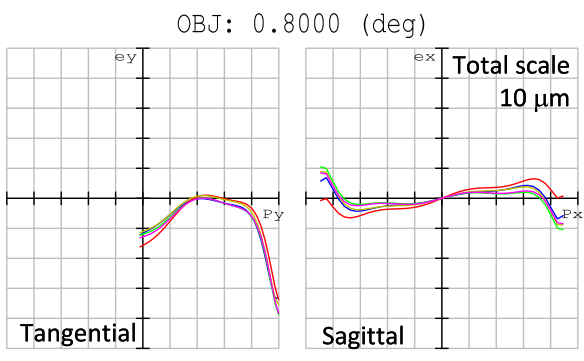

(c)

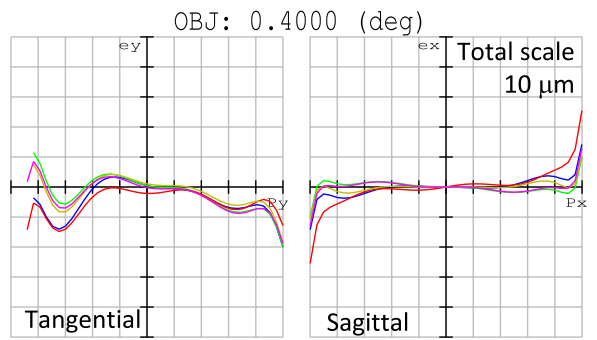

(b)

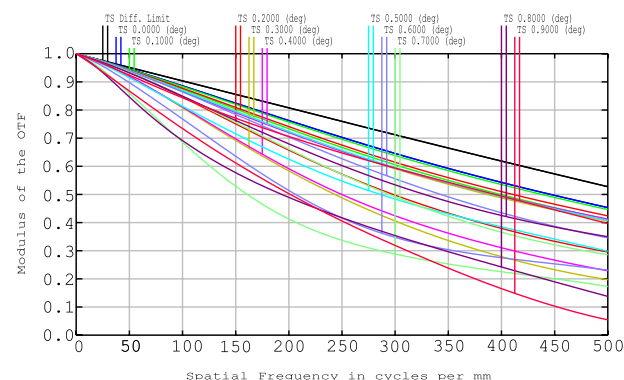

(d)

Fig. 9. (Color online) Ray traces and MTF of $9 \mathrm{~mm}$ aperture, three-element microcamera for the 40 gigapixel objective. (a), (b), and (c) Ray traces for on-axis, half-field, and full-field object points, respectively. (d) Sagittal and tangential modulation transfer functions of this camera as a function of object point. T denotes tangential curves and S denotes sagittal curves in the MTF plot.

similar $\pm 80 \mu \mathrm{m}$ depth-of-field axial tolerance of the microcamera. These tolerances are due to the fact that, if the microcamera is translated or rotated as a whole, the sensitivity is set by the $f / \#$ of the objective and the radius of curvature of the objective image surface. In practice, the tolerances are more likely determined not by image quality but by the need to position the cameras accurately enough to achieve the desired overlap between microcamera fields.

\section{Conclusion}

Multiscale optics ultimately involves the complete integration of the optics at various scales and a corresponding integrated design procedure. While we expect the general design principles and limitations 
to hold, the capabilities might be significantly extended with such a procedure. The joint design and optimization of objectives and microcameras would be a useful study in the future as the chromatic aberration limitations might be alleviated by simultaneous adjustment of glass types in the objective and elements in the microcamera. The current objective designs were created so that their performance could be more easily verified as each forms images, albeit imperfectly, over a particular range of design wavelengths. Independent verification of the objective and microcamera imaging quality allows more conventional optical characterization techniques to be used to diagnose potential problems but is ultimately not necessarily once the right diagnostic toolset is developed for multiscale optical engineering. In short, we have shown how the performance of microcameras for monocentric multiscale systems varies with scale and that a wide range of microcamera scales exists compatible with currentgeneration CMOS sensors and optomechanical fabrication techniques.

This project was supported by the Defense Advanced Research Projects Agency Microsystems Technology Office Advanced Wide Field of View Architectures for Image Reconstruction and Exploitation
(DARPA MTO AWARE) program under contract HR-0011-10-C-0073.

\section{References}

1. D. J. Brady, Optical Imaging and Spectroscopy (Wiley, Optical Society of America, 2009).

2. D. J. Brady and N. Hagen, "Multiscale lens design," Opt. Express 17, 10659-10674 (2009).

3. D. Daly, Microlens Arrays (Taylor \& Francis, 2001).

4. E. H. Adelson and J. Y. A. Wang, "Single lens stereo with a plenoptic camera," IEEE Trans. Pattern Anal. Mach. Intell. 14, 99-106 (1992).

5. M. Levoy, "Light fields and computational imaging," Computer 39, 46-55 (2006).

6. J. E. Ford and E. Tremblay, "Extreme form factor imagers," in Imaging Systems, OSA Technical Digest (CD) (Optical Society of America, 2010), paper IMC2.

7. D. L. Marks and D. J. Brady, "Gigagon: a monocentric lens design imaging 40 gigapixels," in Imaging Systems, OSA Technical Digest (CD) (Optical Society of America, 2010), paper ITuC2.

8. D. Marks and D. Brady, "Close-up imaging using microcamera arrays for focal plane synthesis," Opt. Eng. 50, 033205 (2011).

9. G. J. Swanson, "Binary optics technology: the theory and design of multi-level diffractive optical elements," Tech. Rep. 854 (Massachusetts Institute of Technology, 1989). 